MS29-O2 Long range ordered magnetic and atomic structures of the quasicrystal approximant in the $\mathrm{Tb}-\mathrm{Au}-\mathrm{Si}$ system

Girma Gebresenbut ${ }^{1}$, Mikael Andersson ${ }^{2}$, Přemysl Beran ${ }^{3}$, Pascal Manuel $^{4}$, Per Nordblad ${ }^{2}$, Martin Sahlberg ${ }^{1}$, Cesar Gomez ${ }^{1}$

1. Department of chemistry-Ångström laboratory, Uppsala university, 75121 Uppsala, Sweden

2. Department of Engineering Sciences-Ångström laboratory, Uppsala university, 75121 Uppsala, Sweden

3. Nuclear Physics Institute of the ASCR, v. v. i., Řež 130, 25068 Řež, Czech Republic

4. ISIS Pulsed Neutron and Muon Facility, STFC Rutherford Appleton Laboratory, Chilton, Oxfordshire OX11 0QX, United Kingdom

email: girma.gebresenbut@kemi.uu.se

The atomic and magnetic structure of the 1/1 $\mathrm{Tb}(14) \mathrm{Au}(70) \mathrm{Si}(16)$ quasicrystal approximant has been solved by combining X-ray and neutron diffraction data. The atomic structure is classified as a Tsai-type 1/1 approximant with certain structural deviations from the prototype structures; there are additional atomic positions in the so-called cubic interstices as well as in the cluster centers. The magnetic property and neutron diffraction measurements indicate the magnetic structure to be ferrimagnetic-like below $9 \mathrm{~K}$ in contrast to the related $\mathrm{Gd}(14) \mathrm{Au}(70) \mathrm{Si}(16)$ structure that is reported to be purely ferromagnetic. This is to our knowledge the first reported magnetic structure determination of a quasicrystal approximant with magnetic long-range order

1 Gebresenbut Girma, et. al, Journal of Physics: Condensed Matter 26 (32), 322202 (2014).

Keywords: Quasicrystal approximants, magnetic properties of quasicrystal approximants

\section{MS29-03 Three-dimensional eight-color structure}

\author{
Shelomo I. BEN-ABRAHAM ${ }^{1}$, Dvir FLOM ${ }^{1}$
}

1. Department of Physics, Ben-Gurion University of the Negev, IL-8410501 Beer-Sheba, Israel

email: shelomo.benabraham@gmail.com

We present a three-dimensional generalization of the two-dimensional chair substitution tiling [R1999]. Our structure is a 3D substitution tiling based on eight cubic prototiles labeled $0, \ldots, 7$, or equivalently by eight colors. The structure distinguishes between even and odd sites (labeled by even numbers or warm colors vs. odd numbers or cool colors, respectively). The structure is of the Toeplitz type and hence it has a pure point type Fourier spectrum [BG2013]. We discuss the symmetries of the structure. We also indicate how to generalize the structure to arbitrary dimension.

[BG2013] Baake M. and Grimm U., Aperiodic Order, Volume 1: A Mathematical Cambridge University Press 2013.

[R1999] Robinson E.A., Indag. Mathem. N.S. 10 (1999) 581-599.

Keywords: aperiodic tilings, Toeplitz structure 